\title{
Registro, incorporação tecnológica e produção pública de medicamento: estudo sobre o atazanavir
}

\author{
Registration, technology adoption and public production of a \\ medicine: study of atazanavir
}

\author{
Carla Cristina de Freitas \\ da Silveiral,* \\ Marilena Cordeiro Dias \\ Villela Corrêa' \\ Wanise Borges Gouvea Barroso" \\ Tatiana Aragão Figueiredo"
}

\begin{abstract}
RESUMO
O Brasil foi um dos primeiros países do Sul Global a incluir em suas políticas de saúde pública robustas medidas de controle contra a epidemia de HIV/Aids. Assim, em 2015, cerca de 450 mil pacientes encontravam-se em tratamento no Sistema Único de Saúde. O registro sanitário é uma etapa crucial para entrada do medicamento no mercado nacional, configurando elemento muito favorável ao acesso. Vários setores da sociedade, ainda que baseados em interesses diversos, atuam em prol da incorporação de novas tecnologias que acabam por pesar favoravelmente no controle da epidemia. Nesse trabalho, por meio de um estudo documental descritivo, foi discutido o ciclo do antirretroviral atazanavir, entendido como um ciclo que envolve a seleção do medicamento pelo consenso terapêutico, o registro sanitário e a definição das estratégias de incorporação tecnológica para a produção nacional do medicamento. 0 registro do atazanavir no Brasil foi concedido em 2003, três meses após ter sido obtido no FDA e cinco meses e meio antes de ser concedido na Europa. Esses fatores resultaram em sua rápida inclusão nas recomendações no consenso terapêutico brasileiro, que o adotou como medicamento preferencial ( $1^{\text {a }}$ escolha), em 2004 . Ademais, o atazanavir se tornou objeto de uma parceria público privada para o desenvolvimento do produto, dentro da política hegemônica de incorporação de novas tecnologias em saúde definida pelo Ministério da Saúde, a chamada Política para o Desenvolvimento Produtivo (PDP).
\end{abstract}

PALAVRAS-CHAVE: Registro; Incorporação; PDP; Atazanavir; Saúde Pública

\begin{abstract}
Brazil was one of the first countries in the Global South to include in its public health policies robust control measures against HIV/Aids. Thus, in 2015, approximately 450,000 patients were under treatment by the Unified Health System. The sanitary registration is a crucial step for the drug entry into the domestic market, what configures a very favorable element for the drug access. Several sectors of society, even if based on different reasons, act in favor of the incorporation of new technologies; this fact, ultimately, favorably weighs in controlling the epidemic. In this work the atazanavir antiretroviral cycle is discussed through a descriptive documental study; that cycle involves: the selection of the drug by therapeutic consensus; the sanitary registration; and the definition of technological incorporation strategies for a national drug production. In 2003, the registration of atazanavir was granted in Brazil: only three months after being granted by the FDA in America and only five and a half months before being granted in Europe. This resulted in the rapid inclusion in the recommendations and in a therapeutic consensus being adopted in Brazil as the preferred drug (1st choice) in 2004. Moreover, atazanavir's development became the object of a public-private partnership -known as Productive Development Partnership (PDP)-, inside the hegemonic policy of adopting new health technologies, defined by the Ministry of Health.
\end{abstract}

KEYWORDS: Registration; Adoption; PDP; Atazanavir; Public Health 


\section{INTRODUÇÃO}

No Brasil, a garantia do acesso universal e gratuito ao tratamento antirretroviral (ARV) foi assegurada em novembro de 1996', tendo sido, portanto, um dos primeiros países de renda média a adotar políticas de saúde eficientes e eficazes para o atendimento aos portadores do Vírus da Imunodeficiência Adquirida (HIV). Antes disto, desde o final da década de 1980, já havia se estruturado no país ações do Ministério da Saúde (MS), por meio do Departamento de DST, Aids e Hepatites Virais, para a prevenção da sua transmissão, a qual se seguiram outras ações igualmente importantes como exames de detecção, monitoramento laboratorial da carga viral, genotipagem, entre outras ações de prevenção, tratamento e reabilitação².

Paralelamente à adoção do acesso universal aos ARV, foi instituído o comitê assessor para terapia antirretroviral em adultos e adolescentes, composto por especialistas que se reúnem periodicamente, a fim de definir parâmetros para o tratamento e monitoramento das pessoas vivendo com o HIV/Aids. Os documentos compreendendo as recomendações, gerados pelo referido comitê, são revisados anualmente e conhecidos como consensos terapêuticos ${ }^{3}$, ou seja, os protocolos e diretrizes terapêuticas para o HIV/Aids.

A indicação de início do tratamento é um aspecto que sofreu muitas alterações recentemente, na medida em que os conceitos evoluem; em particular o debate sobre as vantagens do início precoce da triterapia que ampliaram, em muito, o número de pessoas que recebem ARV no Brasil.

A inclusão de um novo ARV no consenso terapêutico só ocorre após a concessão do registro pela Agência Nacional de Vigilância Sanitária (Anvisa) ${ }^{4}$.

As linhas de tratamento do HIV incluem associação de medicamentos, em geral três, de ação terapêutica diferente. Quando um dos medicamentos se mostra contraindicado a um paciente individual, ele deve ser substituído por outro da mesma classe ou deve ser indicada uma associação diversa, com mudança de dois ou de três medicamentos do chamado coquetel ARV 5 .

Simultaneamente à avaliação clínica e laboratorial dos pacientes que indica a necessidade de mudanças de ARV, seja por intolerância ao uso, efeitos colaterais ou resistência, a pressão para incorporação de novas tecnologias farmacológicas se dá tanto pelo mercado quanto por parte das associações de pacientes e profissionais da área da saúde ${ }^{6}$.

Atualmente, a incorporação de tecnologias em saúde no âmbito do Sistema Único de Saúde (SUS) é responsabilidade da Comissão Nacional de Incorporação de Tecnologias no SUS $(\text { CONITEC })^{7,8}$. Os medicamentos ARV fazem parte do chamado Componente Estratégico da Assistência Farmacêutica, que possui financiamento e aquisição centralizados pelo MS em uma logística própria de funcionamento ${ }^{9}$. A CONITEC assessora o MS na incorporação, exclusão ou alteração pelo SUS de tecnologias em saúde. Atua também na definição e alteração de Protocolos Clínicos e Diretrizes Terapêuticas (PCDT), conforme o interesse para o SUS. Nesse processo, a Avaliação Tecnológica em Saúde (ATS) é utilizada e objetiva auxiliar a tomada de decisão de forma crítica, fundamentada na melhor evidência farmacológico-clínica ${ }^{10}$. Para avaliação da substituição de dada tecnologia, a CONITEC leva em consideração aspectos como eficácia, acurácia, efetividade e segurança da tecnologia, além da avaliação econômica comparativa dos benefícios e custos, em relação às tecnologias já existentes ${ }^{11}$.

O governo brasileiro, no afã de reduzir o déficit na área da saúde e fomentar a pesquisa, desenvolvimento e inovação tecnológica no país, tem proposto ações e subsidiado investimentos nos setores que compõem a tríade universidade, empresas e governo. Faz parte dessa política, propor e rever periodicamente a lista dos produtos estratégicos no âmbito do SUS, definida pelo MS, na qual se incluem os $A R V^{12}$.

A lista de produtos estratégicos para o SUS é a base da priorização dos projetos de parceria de desenvolvimento produtivo (PDP), sendo que as PDP são contratos firmados entre instituições públicas e entidades privadas com vistas à transferência e posterior absorção de tecnologia de produção de produtos estratégicos para o SUS ${ }^{13}$. Espera-se, no médio prazo, que a experiência das PDP eleve a capacidade produtiva e a capacitação tecnológica do país.

Da lista de medicamentos estratégicos do MS, que deverão vir a ser produzidos nacionalmente, constam 15 dos 26 ARV presentes na Relação Nacional de Medicamentos Essenciais (Rename) de 2014 14,15,16. Entre eles, o atazanavir, que será detalhadamente abordado na segunda parte deste artigo.

O atazanavir foi objeto da primeira PDP de ARV formalizada entre o ente público Farmanguinhos/Fiocruz, a farmoquímica nacional Nortec e a parceira privada Bristol-Myers Squibb (BMS). A formalização desta PDP se deu em novembro de 2011, encontrando-se hoje em fase de desenvolvimento industrial, com a transferência de tecnologia e a integração gradativa do processo produtivo do medicamento e de seu insumo farmacêutico ativo (IFA) que ficará a cargo da Nortec $^{17}$, enquanto Farmanguinhos produzirá o medicamento final.

O estudo do ciclo do atazanavir possibilitará acompanhar as fases de incorporação da tecnologia do fármaco, a produção do medicamento e sua distribuição aos usuários, dentro das políticas farmacêutica, de saúde pública e de fortalecimento do complexo médico-industrial da saúde no país.

Neste contexto, o presente trabalho objetiva analisar o ciclo do atazanavir que inclui o registro sanitário, a incorporação, a seleção como medicamento estratégico para a produção nacional e o estabelecimento da PDP. 


\section{MÉTODO}

Trata-se de um estudo documental descritivo.

Fonte de dados

Para o registro sanitário na Anvisa, foi consultada a base de dados da agência, no item "Registro de Medicamentos" (http://www. anvisa.gov.br). E ainda o Orange Book, disponível no sítio do US Food and Drug Administration (FDA) (http://www.fda.gov), para consulta dos registros concedidos nos Estados Unidos, e o sítio da Agência Europeia de Medicamentos (European Medicines Agency - EMA) (http://www.ema.europa.eu), para consulta dos registros concedidos na Europa.

A pesquisa e coleta nas bases de dados foram realizadas entre dezembro/2015 e fevereiro/2016.

Para a consulta dos documentos oficiais do MS referentes ao tratamento de HIV/Aids, utilizou-se o sítio do Departamento de DST, Aids e Hepatites Virais (http://www.aids.gov.br).

Para identificação da lista dos medicamentos estratégicos para produção nacional, foi consultada a Portaria $n^{\circ} 3.089$, de 11 de dezembro de $2013^{18}$.

Os dados referentes às PDP de ARV foram obtidos a partir do sítio do Departamento do Complexo Industrial e Inovação em Saúde no Portal da Saúde (http://portalsaude.saude.gov.br/).

O Acordo referente à PDP foi obtido através do sítio do Grupo de Trabalho sobre Propriedade Intelectual ${ }^{19}$ (http://www.deolhonaspatentes.org.br/).

Plano de análise

As bases de dados oficiais da Anvisa, FDA e EMA foram consultadas a fim de identificar: 1) datas de registro; 2 ) empresas requerentes do registro e 3 ) apresentações farmacêuticas referentes ao atazanavir.

Os documentos do MS foram consultados a fim de identificar a inclusão do medicamento atazanavir no tratamento recomendado para HIV/Aids. Tal proposta visou identificar o tempo entre o registro sanitário e a incorporação do medicamento pelo SUS.

A partir do sítio do Departamento do Complexo Industrial e Inovação em Saúde e do acordo da PDP, obtiveram-se informações referentes a: 1) ano de aprovação da proposta de projeto da PDP; 2) objetivos; 3) atores envolvidos na parceria e 4) fase de desenvolvimento da PDP que divide-se em fase I (Propostas de Projeto de PDP), fase II (Projetos de PDP), fase III (PDP) e fase IV (Internalização da Tecnologia).

\section{RESULTADOS E DISCUSSÃO}

O acesso a medicamentos para tratamento da Síndrome da Imunodeficiência Adquirida (Aids) no Brasil é prioridade inserida na agenda política do Estado e é essencial para a política nacional de saúde.
O estudo do ciclo do ARV atazanavir permite uma reflexão acerca da escolha do atazanavir para transferência de tecnologia via PDP.

\section{Registro do atazanavir na Anvisa, FDA e EMA}

De acordo com a legislação brasileira, os medicamentos de procedência estrangeira devem, além do registro no Brasil, obrigatoriamente ter comprovação do registro no país de origem ${ }^{20}$. A empresa interessada em registrar um novo medicamento no Brasil deve submeter à Agência um dossiê detalhado que subsidiará a análise farmacotécnica e as análises de eficácia e segurança.

No ato do pedido de registro à Anvisa para novos medicamentos, as empresas devem apresentar também relatório com a previsão do preço. Caso o medicamento já tenha sido comercializado em outros países, a base será o preço praticado nesses locais. A aprovação final ficará condicionada à previsão de preço a ser praticado no mercado ${ }^{21}$.

O FDA, agência reguladora de medicamentos dos Estados Unidos, é, em muitas situações, tomada como modelo de órgão regulador e principal referência mundial para aprovação e introdução de um novo medicamento no mercado. 0 processo de registro de medicamento no FDA inclui a avaliação dos ensaios clínicos em seres humanos, a comprovação da segurança e eficácia para o uso e indicação pretendidos, a toxicidade, a certeza de que seus benefícios compensam os riscos, assim como a garantia de que a forma como o medicamento será produzido irá manter as características originais do novo medicamento aprovado. Após o exame dos dados científicos apresentados, são realizados testes para identificar a veracidade das informações fornecidas pelo fabricante do medicamento ${ }^{22,23}$.

Depois do FDA, a EMA é considerada o mais importante órgão mundial que avalia os dados científicos apresentados pelos fabricantes de novos medicamentos. A EMA é responsável pela análise dos pedidos de autorização de introdução no mercado de medicamentos dos 30 países da União Europeia. É apresentado um único pedido de autorização de introdução no mercado junto à EMA, válido para todos os países-membros. 0 solicitante do registro do medicamento na EMA é obrigado a fornecer os dados sobre a comercialização em outros países, qualquer resultado novo ou diferente sobre a segurança do medicamento e sobre ações regulatórias relacionadas a sua segurança. Deverão ser apresentadas também as conclusões sobre os riscos e benefícios do medicamento, quando ele for comparado com alternativas farmacológicas disponíveis para tratamento da doença ou com nenhum tratamento farmacológico, quando esta for uma opção clinicamente aceitável ${ }^{24}$

O controle dos medicamentos é realizado por meio de diferentes formas pelas autoridades regulatórias dos países e a exigência do registro sanitário prévio à comercialização compreende uma das medidas fundamentais para a proteção e acesso à saúde da população. As informações oficiais disponíveis nas agências apresentam diferenças em termos quantitativos e qualitativos, o mesmo ocorre com a definição do que é considerado novo medicamento pelas autoridades sanitárias ${ }^{23,25}$. 
No Quadro são apresentados os registros do ARV atazanavir na Anvisa, FDA e EMA e as respectivas empresas requerentes. Foram concedidos registros para apresentação de 100, 150, 200 e 300 mg.

De acordo com a busca realizada no sítio da Anvisa, a BMS e a Fundação Oswaldo Cruz (Fiocruz) possuem registro válido para o sulfato de atazanavir. A BMS solicitou o registro em março de $2003^{4}$ e sua concessão ocorreu seis meses depois, em 18 de setembro de 2003.

A concessão do registro do medicamento sulfato de atazanavir para a Fiocruz ocorreu em 13 de janeiro de 2014 e compreende uma das etapas do processo de PDP, referente à cooperação técnico-científica entre a Fiocruz, representada por Farmanguinhos e a BMS, a qual visa à transferência de tecnologia do medicamento atazanavir e a produção do respectivo IFA, no Brasil.

Em dezembro de 2002, a BMS solicitou ao FDA o registro do atazanavir nos Estados Unidos. Em 20 de junho de 2003, seis meses após a solicitação, o primeiro registro do atazanavir foi concedido. Em 20 de outubro de 2006, a BMS conseguiu aprovação para nova formulação de atazanavir ( 300 mg cápsulas) utilizado como parte da terapia de combinação de fármacos, que reduz a carga de comprimidos, pois pode substituir duas cápsulas de $150 \mathrm{mg}$.

Em 22 de abril de 2014, a empresa israelense Teva Pharms USA também conseguiu aprovação para o seu registro no FDA. Entretanto, só poderá comercializar o seu genérico após a expiração da patente do atazanavir, em 2017.

Em 22 de abril de 2002, a BMS submeteu solicitação de registro do atazanavir na EMA, que foi concedido 23 meses após a solicitação, em 2 de março de 2004.

Utilizando como referência o FDA, que geralmente concede o primeiro registro do medicamento, é possível comparar o tempo entre o registro na agência norte-americana e nas agências brasileira e europeia.

O Brasil foi o segundo país do mundo a conceder o registro do atazanavir. A sua solicitação de registro aqui no país foi realizada antes mesmo de ter sido concedido o registro nos Estados Unidos, e culminou com a concessão do registro logo após três meses do registro no FDA ${ }^{4}$. Um tempo muito inferior se compararmos a diferença entre os registros nas agências FDA e EMA,

Quadro. Data de concessão do registro do atazanavir na Anvisa, FDA e EMA.

\begin{tabular}{|lcc|}
\hline Atazanavir & $\begin{array}{c}\text { Data de concessão do } \\
\text { registro }\end{array}$ & $\begin{array}{c}\text { Empresa } \\
\text { requerente }\end{array}$ \\
\hline \multirow{2}{*}{ Anvisa } & $18 / 09 / 2003$ & BMS \\
& $13 / 01 / 2014$ & Fiocruz \\
FDA & $20 / 06 / 2003$ & BMS \\
& $22 / 04 / 2014$ & Teva Pharms USA \\
EMA & $02 / 03 / 2004$ & BMS \\
\hline
\end{tabular}

FDA: US Food and Drug Administration; EMA: European Medicines Agency; BMS: Bristol-Myers Squibb; Fiocruz: Fundação Oswaldo Cruz Fonte: Elaboração própria a partir de dados extraídos do sítio da Anvisa, FDA e EMA. que foi de oito meses e meio. o curto espaço de tempo para a concessão do registro no Brasil exemplifica um caso de medicamento que, após o registro no FDA, foi rapidamente incluído nas recomendações no consenso terapêutico no Brasil.

Quando comparado o tempo de tramitação entre o registro na Anvisa e na EMA, observa-se que no Brasil o registro foi concedido cinco meses e meio antes do registro na Europa.

É mister pontuar que o registro pela autoridade sanitária permite a comercialização do medicamento, independentemente do proponente ser um produtor público ou privado, não estando relacionado ao posterior processo de incorporação do mesmo no sistema de saúde. Por outro lado, a produção pública do medicamento por Laboratório Farmacêutico Oficial (LFO) requer o registro sanitário e estes laboratórios devem produzir medicamentos já incorporados no sistema, uma vez que buscam atender às demandas do MS e das Secretarias de Saúde. Neste sentido, o lapso temporal para a obtenção do registro por parte dos LFO não está relacionado à incorporação do medicamento, mas sim à política industrial por meio do Complexo Econômico-Industrial da Saúde com vistas à ampliação do acesso de medicamentos estratégicos à população.

As exigências quanto ao tipo e volume de informações que devem ser fornecidas pelos solicitantes de registro às autoridades sanitárias e os prazos legais para análise da solicitação de registro também são distintas em cada país ${ }^{26}$. Entretanto, o registro sanitário de medicamentos configura um importante instrumento de regulação sanitária por meio do qual o Estado deve atuar como mediador entre os interesses das empresas produtoras de medicamentos, que desejam registrar seus produtos e os interesses da saúde pública, zelando por sua defesa e proteção.

O registro precoce ou tardio, com espaços de tempo discrepantes pode ser decorrente de uma avaliação deficiente do impacto do medicamento no tratamento dos pacientes e no sistema de saúde. Para atender ao interesse da saúde pública é essencial que os trâmites, as regras e os parâmetros de regulação sejam mais transparentes para todos os envolvidos no sistema.

\section{Incorporação do atazanavir no SUS}

Os PCDT são documentos oficiais do SUS que estabelecem os critérios para o diagnóstico de uma doença ou agravo à saúde; o tratamento preconizado, com os medicamentos e demais produtos apropriados, quando couber; as posologias recomendadas; os mecanismos de controle clínico; e o acompanhamento e a verificação dos resultados terapêuticos a serem seguidos pelos gestores do SUS ${ }^{27}$.

Os medicamentos ou produtos recomendados no PCDT incluem as diferentes fases evolutivas da doença ou do agravo à saúde, bem como aqueles indicados em casos de perda de eficácia e de surgimento de intolerância ou reação adversa relevante, provocadas pelo medicamento, produto ou procedimento de primeira escolha. Para elaborar os PCDT, considera-se a análise baseada em evidências científicas, levando-se em consideração os critérios de eficácia, segurança, efetividade e custo-efetividade das 
intervenções em saúde recomendadas. Para garantir a disponibilização das tecnologias previstas no PCDT e incorporadas ao SUS, a lei estipula um prazo de 180 dias para a efetivação de sua oferta à população brasileira. Os PCDT possuem um caráter normativo e não apenas orientações a serem seguidas.

Após consulta ao sítio do Departamento de DST, Aids e Hepatites Virais, foi verificado que os PCDT no formato de protocolo para HIV/Aids e para as demais doenças oportunistas ou relacionadas foram publicados após 2014. Nos anos anteriores, as publicações eram denominadas Recomendações-Consensos de Terapia e reuniam técnicas de especialistas de todo o país para tratamento de soropositivos.

O atazanavir foi incorporado em 2004 como medicamento preferencial, de $1^{\text {a }}$ escolha, no consenso Recomendações para Terapia Antirretroviral em Adultos e Adolescentes Infectados pelo HIV $2004^{28}$.

Em outubro de 2003, o Programa Nacional de DST e Aids do MS reuniu o Comitê Assessor com o objetivo de discutir os recentes avanços na área e assistir o Programa na revisão das estratégias para o tratamento da infecção pelo HIV em adultos e adolescentes. Nesta revisão, foram considerados todos os medicamentos ARV registrados e disponíveis para uso clínico no país até a data da última reunião do comitêt ${ }^{28}$.

Através do referido consenso, foram definidas para o atazanavir as condutas clínicas e terapêuticas consideradas seguras e eficazes à luz do conhecimento médico-científico da época, baseado em resultados de estudos clínicos metodologicamente válidos, publicados em revistas científicas ou apresentados em congressos e as condutas pouco avaliadas ou danosas ao paciente e que não deveriam ser utilizadas. A inclusão do atazanavir como medicamento preferencial foi baseada na facilidade posológica e no bom perfil de toxicidade.

Em outubro de 2005, após nova reunião, foi publicado o consenso Recomendações para Terapia Antirretroviral em Adultos e Adolescentes Infectados pelo HIV 2005/2006. Neste consenso, o atazanavir potencializado com ritonavir (ATV/r) foi considerado como opção preferencial. A sua inclusão foi baseada na tendência mundial e em estudos que apontam para menor risco de falha e de mutações de resistência com esquemas iniciais que incluem inibidores de protease (IP) potencializados com ritonavir (IP/r); quando comparados com aqueles que incluem IP sem potencialização, incluindo estudo comparativo com o próprio medicamento. $O$ atazanavir não potencializado pelo ritonavir passa a ser segunda opção de IP. A opção de atazanavir sem ritonavir foi mantida, considerando-se os resultados favoráveis de estudos de atazanavir sem reforço para pacientes virgens de tratamento e, particularmente, o fato de que alguns indivíduos não toleram ritonavir mesmo na dose baixa de $100 \mathrm{mg}$ por dia ${ }^{29}$.

O atazanavir também se mostra presente em listas de medicamentos de outros países que contam com sistemas universais de saúde, como Reino Unido e Austrália. Na lista do Sistema Nacional de Saúde do Reino Unido (NHS - National Health System), o atazanavir é indicado para tratamento do HIV/Aids, mas não há especificação da linha de tratamento. E a lista de medicamento do sistema de saúde australiano (The Pharmaceutical Benefits Scheme - PBS) possui várias apresentações do atazanavir ${ }^{30,31}$.

Em consulta ao Thomson Micromedex - Drugdex System, verifica-se que o uso do atazanavir em tratamento de HIV/Aids é considerado efetivo. No que tange a força da evidência científica, o atazanavir apresenta nível A (mais alto nível), ou seja, evidência baseada em metanálises de estudos clínicos randomizados controlados homogêneos, com desfechos e graus de resultados entre os estudos individuais. Por fim, o medicamento possui força de recomendação Classe lla ou seja, segundo a classificação da base Thomson, este medicamento é considerado útil para o tratamento de HIV/Aids e apresenta indicação para a maioria dos $\operatorname{casos}^{32}$.

0 atazanavir mostra-se um medicamento sólido para o tratamento de HIV/Aids, com forte evidência científica e presença em outras listas de medicamentos de sistemas de saúde de outros países. A escolha do mesmo como estratégico para o SUS, ou seja, para a produção nacional mostra-se coerente frente à força do medicamento e à importância do programa nacional de DST e Aids para o SUS.

\section{A produção pública do atazanavir via PDP}

A escolha do atazanavir como medicamento no SUS é coerente. No entanto, a sua produção pública, no Brasil, por meio da PDP, é uma questão a ser discutida.

Para assegurar o acesso ao ARV atazanavir, o governo adotou como estratégia o processo de incorporação da tecnologia no sistema de saúde, via PDP para garantir a disponibilidade, a continuidade e expansão do tratamento, a redução de preço e os esforços para a produção local.

Através da PDP para a fabricação do atazanavir, firmada em 11 de novembro de 2011 entre Farmanguinhos/Fiocruz e BMS, o governo iniciou o processo de transferência de tecnologia para a produção do medicamento no país e a sua internalização para atendimento das demandas do SUS.

A BMS, titular da patente do atazanavir, possui direito de exclusividade sobre a comercialização do medicamento no Brasil até 2017. Conforme argumenta Chaves et al. ${ }^{33}$, quando um novo medicamento é inserido no consenso terapêutico e é patenteado, o governo fica obrigado por lei a garantir o acesso a ele. Assim, a empresa tem posição vantajosa, pois sabe que o governo será obrigado a comprar o medicamento, ainda que os preços sejam altos.

Na PDP do atazanavir, está prevista a licença voluntária da patente vigente e transferência de tecnologia do IFA e das formas farmacêuticas em cápsulas de $200 \mathrm{mg}$ e $300 \mathrm{mg}$. Entretanto, a redução de preços esperada pelo governo pode não ser alcançada e o que se observará, na prática, será uma extensão do monopólio patentário por atrasos na execução da parceria.

O não cumprimento do prazo do processo de transferência de tecnologia a Farmanguinhos, associado ao atraso de 18 meses na 
concessão do registro sanitário, obtido em janeiro de 2014, assegura à BMS mercado, mesmo após a expiração da patente, uma vez que Farmanguinhos está obrigado a comprar e pagar royalties por cinco anos após a obtenção do registro sanitário. A BMS suprirá $100 \%$ da demanda do MS nos três primeiros anos após a concessão do registro sanitário e $50 \%$, no quarto e quinto ano $^{19}$.

Para a BMS, a assinatura da PDP do atazanavir, próxima ao prazo de expiração da patente, dentro do contexto de atraso na internalização da tecnologia e na finalização da PDP, se traduz em ganho real para aquela empresa, com oportunidade de exploração da patente além do tempo previsto.

Outro ponto importante a ser discutido, e que não foi contemplado no contrato da PDP do atazanavir, é a inclusão dos pedidos de patentes, que sejam posteriores a patente principal e que conferem proteção relacionada ao medicamento. É necessário que, além das patentes já concedidas, sejam informados também eventuais pedidos de patente que estejam pendentes de análise que se relacionam com o produto objeto da PDP. Uma patente concedida posteriormente pode tornar a tecnologia a ser desenvolvida pela PDP obsoleta ou bloquear a sua produção nacional.

É essencial que, no caso de produtos que estejam patenteados ou com pedidos de patentes pendentes, as referidas informações sejam avaliadas e consideradas quando da negociação da PDP, de forma a não limitar a possibilidade de produção pelo Brasil.

A inclusão do atazanavir no acordo da PDP apenas como monofármaco também é fator relevante e que merece atenção. O contrato apresenta cláusula limitante para produção de apenas uma apresentação farmacêutica do medicamento (cápsula $200 \mathrm{mg}$ e $300 \mathrm{mg}$ ), excluindo expressamente a produção de qualquer outra apresentação ou combinação do atazanavir. No entanto, em recente orientação, a Organização Mundial da Saúde (OMS) recomendou a utilização da combinação do atazananir com o ritonavir ${ }^{34}$. Essa combinação já está sendo utilizada por outros países, tendo em vista seus benefícios para os portadores de $\mathrm{HIV}^{35}$.

A tecnologia prevista no contrato assinado no Brasil não está de acordo com as tendências globais de tratamento, que prevê a indicação do atazanavir associado a outro ARV, o ritonavir, que potencializa o seu efeito, além de facilitar a adesão ao tratamento. No contrato de transferência assinado entre a BMS e o Medicines Patent Pool (MPP), para aumentar o acesso ao atazanavir em países em desenvolvimento, tal proibição não existe e caberá ao licenciado o direito de combinar atazanavir com outros ARV e desenvolver novas combinações de dose fixa ${ }^{36}$.

Considerando os apontamentos, pode ser visto que o Brasil corre o risco de finalizar o processo de transferência do atazanavir, produzindo um medicamento que poderá ser substituído em breve e que pode não atender às recomendações mundiais de autoridades em saúde pública. Em virtude da restrição contratual, pelo menos até 2019, o Brasil não poderá produzir essa combinação, mesmo possuindo tecnologia para tanto, em razão de uma limitação imposta pela empresa BMS no contrato da PDP ${ }^{19}$.
Analisando as estratégias que poderiam ter sido utilizadas pelo governo para a produção do atazanavir, cabe a reflexão se a PDP foi a melhor opção. E quais outras opções poderiam ter sido adotadas para garantir a produção local e o acesso universal ao medicamento.

Entre as estratégias já utilizadas pelo governo, destacam-se a política de medicamentos genéricos, com investimento em laboratórios nacionais para produção de versões genéricas dos ARV que entram em domínio público; negociações de preços dos produtos patenteados por meio de acordos de troca de tecnologia com os laboratórios ainda detentores das patentes e a incorporação de salvaguardas do Acordo TRIPS (Acordo sobre Aspectos dos Direitos de Propriedade Intelectual Relacionados ao Comércio) para proteção da saúde pública.

De forma a alcançar o acesso universal ao tratamento e garantir o fornecimento de ARVs, o governo brasileiro utilizou como primeira grande estratégia a produção nacional dos medicamentos que não estavam protegidos por patentes, inventados antes de 1997 (patenteados no exterior, mas não no Brasil, que não reconhecia patentes farmacêuticas até 1996) e que puderam ser livremente estudados, copiados e produzidos. A cópia dos medicamentos ARV, por meio de engenharia reversa, foi fundamental para o sucesso da política de acesso universal ao tratamento para HIV/Aids e constituiu um modo particular de aquisição de conhecimentos, aprendizado tecnológico e difusão local da tecnologia ${ }^{37}$.

Apesar de a referida estratégia não poder ser aplicada para o caso do atazanavir, já que possui proteção patentária, sua avaliação e os ganhos adquiridos com a sua implementação devem ser considerados, pois refletem a importância de investimento em pesquisa e desenvolvimento (P\&D) nos LFO, de forma a capacitá-los para reproduzir ou até mesmo melhorar os produtos patenteados, ainda que em escala laboratorial.

Conforme bem apontado por Chaves $^{38}$, o desenvolvimento local do medicamento até a obtenção do registro sanitário pode ser utilizado para estimar custos de produção e subsidiar negociação de preços entre o MS e a empresa detentora da patente do medicamento, além de habilitar o uso da licença compulsória quando essas negociações não forem favoráveis. A implantação da política atual de transferência de tecnologia do atazanavir por meio da PDP compreende um processo totalmente oposto ao apresentado na estratégia citada, visto que, na PDP, não cabe otimização de produção por parte dos laboratórios que estão recebendo a tecnologia. O Brasil necessita desenvolver competências em P\&D tecnológico no setor farmacêutico como meio de garantir ao país níveis de produção interna de medicamentos compatíveis com a demanda e reduzir a crescente dependência das importações de insumos, know-how e medicamentos.

Para os medicamentos ARV de segunda e terceira geração, protegidos por patentes não passíveis de cópia e produção local, uma das estratégias adotada pelo governo foi pressionar os laboratórios detentores dos direitos de propriedade a reduzir o preço dos produtos, sob ameaça de licença compulsória das patentes, prevista na Lei de Propriedade Industrial brasileira ${ }^{39}$. 0 licenciamento compulsório pode não ser a melhor medida adotada por 
um governo para garantir o acesso de sua população a medicamentos essenciais. Entretanto, em virtude da falta de interesse das empresas em licenciar voluntariamente tecnologias de produção de fármacos, esta seria uma solução a ser avaliada.

No caso do atazanavir, caso a licença compulsória fosse decretada, na mesma data o país seria autorizado a produzir o medicamento, porém, a sua capacidade produtiva poderia ser mais demorada; diferentemente da transferência via PDP, na qual são previstos todo apoio tecnológico e instruções necessárias para o desenvolvimento do produto. Enquanto o país não fosse suficiente na produção, teria de comprar o fármaco na versão genérica de outro país.

Antes de negociar uma licença voluntária da patente, através de uma PDP, deve-se considerar a possibilidade de utilização de salvaguardas como: subsídio ao exame (oposição), diretrizes de exame restritivas na interpretação dos requisitos de patenteabilidade, atuação do setor saúde no processo de exame de pedidos de patente (Anuência - Anvisa), importação paralela, licença compulsória, entre outras ${ }^{40}$.

No único caso de emissão da licença compulsória, por interesse público, no Brasil, emitida em 2007 para o antirretroviral efavirenz utilizado no tratamento de HIV/Aids, o Brasil economizou $58,47 \%$ em compras de genéricos no período de cinco anos, entre 2007 e 2011. E o tempo necessário para a produção nacional do medicamento foi de 22 meses depois da licença compulsória, mesmo sem a transferência de tecnologia pelo detentor da patente ${ }^{38}$. Com a chegada ao mercado do genérico do efavirenz produzido no Brasil, houve uma redução de preço de $93 \%$ e os gastos caíram para $3,9 \%$ no país ${ }^{41}$.

A comparação entre os preços pagos no Brasil pelo atazanavir em relação ao menor preço ofertado pela BMS (preço de desconto) e o da versão genérica mostrou que aqueles praticados no país são os mais altos. No entanto, essas alternativas não puderam ser utilizadas pelo Brasil, uma vez que o país não constava na relação dos países beneficiados pela política de discriminação de preços da BMS e também porque as empresas produtoras da versão genérica eram licenciadas pela BMS e as referidas licenças se restringem à comercialização em países da África e Índia ${ }^{33}$.

Uma análise preliminar da PDP do atazanavir realizada por Chaves $^{38}$ releva que, na estratégia utilizada para reduzir preços, envolvendo produção local da cápsula de $200 \mathrm{mg}$, as reduções foram menores do que os $5 \%$ estimados no acordo. Quanto à cápsula de $300 \mathrm{mg}$, os valores pagos nos dois primeiros anos após a PDP foram próximos aos estimados. Os preços em valores nominais mantiveram-se praticamente constantes entre 2011 (ano da assinatura da PDP), 2012 e 2013 (depois de estabelecida a Par(ceria) $)^{38}$. A produção nacional ainda não foi iniciada, mesmo com a transferência de tecnologia realizada pela BMS, detentora da patente. E a garantia de exclusividade de mercado ao detentor da patente vai perdurar por cinco anos adicionais após o registro do produto nacional, fato que não ocorreu no caso de licença compulsória do efavirenz, em que o produto nacional pode ser utilizado imediatamente pelo SUS.
Os resultados obtidos nas estratégias citadas mostram que, independentemente da estratégia utilizada pelo governo, o objetivo final deve ser a produção nacional, sendo esta a única maneira de garantir o abastecimento do ARV para o SUS e permitir o maior controle sobre sua distribuição, já que não dependerá de laboratório estrangeiro. Os ganhos para o Brasil e para o fortalecimento da política nacional de acesso a medicamentos devem ir além da economia de recursos financeiros, aumentar a credibilidade do governo para negociar preços de outros produtos farmacêuticos e estimular o fortalecimento da produção nacional e também a transferência de tecnologia.

\section{CONCLUSÃO}

O registro do ARV atazanavir no Brasil foi concedido em 2003, logo após três meses do registro obtido no FDA e cinco meses e meio antes do registro ser concedido na Europa.

É importante destacar que este estudo não tem como propósito realizar uma análise de comparação de desempenho das agências reguladoras, mas sim ilustrar o tempo para obtenção de registro do atazanavir nas três agências citadas.

Em 2004, o ARV atazanavir foi incorporado como medicamento preferencial, de $1^{\text {a }}$ escolha e, em 2005, o ATV/r foi considerado como opção preferencial, com base em estudos que apontam para menor risco de falha e de mutações de resistência com esquemas iniciais que incluem IP/r. Ele compõe também a lista de medicamentos de outros países com sistema de saúde universal, como Reino Unido e Austrália, e a sua escolha como estratégico para o SUS mostra-se coerente frente à força do medicamento e à importância para o programa nacional de DST e Aids.

Os elevados preços de aquisição dos medicamentos ARV constituem a principal barreira de acesso e um dos principais desafios do programa brasileiro de combate à Aids. E caberá ao governo estabelecer a política mais adequada para garantir a sua produção local.

O MS, enquanto comprador de medicamentos, deve buscar estratégias de equilíbrio entre a incorporação de novos medicamentos no SUS e a implementação de políticas específicas que conduzam a ampliação do acesso aos medicamentos para o tratamento da Aids no Brasil.

A cláusula restritiva presente no contrato da PDP do atazanavir que proíbe expressamente a sua fabricação, na forma combinada ou em apresentações, dosagens, formulações e composições que sejam diferentes da forma de cápsula acabada nas apresentações de $200 \mathrm{mg}$ e $300 \mathrm{mg}$, é um fator limitador para o acesso às novas formulações contendo atazanavir, que possivelmente serão recomendadas e usadas como novas opções de tratamento.

O atraso não previsto no contrato da PDP para obtenção do registro sanitário por Farmanguinhos junto à Anvisa acabou por assegurar à BMS a extensão do monopólio conferido pela patente e o pagamento de royalties mesmo após a sua expiração.

No caso de produtos que estejam protegidos por patente ou com pedidos de patentes pendentes, é crucial que as referidas 
informações sejam avaliadas e consideradas quando da negociação da PDP, de forma a não limitar a possibilidade de produção pelo país.

Para alcançar a sustentabilidade através do incentivo à produção local, o Brasil necessita desenvolver competências em P\&D e tecnologias no setor farmacêutico, como meio de garantir ao país níveis de produção interna de medicamentos compatíveis com a demanda, com redução crescente da dependência das importações de insumos, know-how e medicamentos. Expandir a produção nacional deve ser a prioridade para o alcance desses objetivos.
A experiência adquirida com o processo de PDP, atrelada a outras políticas públicas já adotadas pelo governo, pode ser aplicada para acelerar os mecanismos para produção de fármacos no país, garantir ao governo o poder de regular os preços, criar uma política mais articulada para analisar os critérios relevantes para os acordos de transferência de tecnologia e atrair parceiros internacionais, prevenir os gastos com ARV, aumentar o acesso dos ARV à população e, consequentemente, garantir a sustentabilidade do programa de distribuição universal de agentes ARV pelo SUS.

\section{REFERÊNCIAS}

1. Brasil. Lei $\mathrm{n}^{\circ} 9.313$, de 13 de novembro de 1996. Dispõe sobre a distribuição gratuita de medicamentos aos portadores do HIV e doentes de AIDS. Diário Oficial União. 14 nov 1996.

2. Loyola MA. Aids e saúde pública: a implantação de medicamentos genéricos no Brasil. In: Corrêa MCDV, Cassier $M$, editores. Aids e saúde pública: contribuições à reflexão sobre uma nova economia política do medicamento no Brasil. Rio de Janeiro: EdUERJ; 2010. p 17-70.

3. Ministério da Saúde (BR), Secretaria de Vigilância em Saúde, Programa Nacional de DST e Aids. Recomendações para terapia antirretroviral em adultos infectados pelo HIV 2008. 7a ed. Brasília, DF: Ministério da Saúde; 2008.

4. Scheffer MC. Aids, tecnologia e acesso sustentável a medicamentos: a incoporação dos anti-retrovirais no Sistema único de Saúde [tese]. São Paulo: Universidade de São Paulo; 2008.

5. Ministério da Saúde (BR). Protocolo clínico e diretrizes terapêuticas para manejo da infecção pelo HIV em adultos. Brasília, DF: Ministério da Saúde; 2013[acesso 10 out 2015]. Disponível em: http://www.aids.gov.br/pcdt/ protocolo-clinico

6. Conselho Nacional de Secretários de Saúde - Conass. Ciência e tecnologia em saúde. Brasília, DF: Conass; 2011. (Coleção Para entender a gestão do SUS, vol 11).

7. Brasil. Lei $n^{\circ} 12.401$, de 28 de abril de 2011. Altera a Lei $\mathrm{n}^{\circ} 8.080$, de 19 de setembro de 1990 , para dispor sobre a assistência terapêutica e a incorporação de tecnologia em saúde no âmbito do Sistema Único de Saúde - SUS. Diário Oficial União. 29 abr 2011.

8. Brasil. Decreto $n^{\circ} 7.646$. de 21 de dezembro de 2011. Dispõe sobre a Comissão Nacional de Incorporação de Tecnologias no Sistema Único de Saúde e sobre o processo administrativo para incorporação, exclusão e alteração de tecnologias em saúde pelo Sistema Único de Saúde - SUS, e dá outras providências. Diário Oficial União. 22 dez 2011.

9. Silva RM. Programa "Aqui tem Farmácia Popular": gastos entre 2006 - 2012 e comparação com os custos da assistência farmacêutica na Secretaria Municipal de Saúde do Rio de Janeiro [tese]. Rio de Janeiro: Universidade do Estado do Rio de Janeiro; 2014.

10. Oliveira MA, Bermudez JAZ, Osorio-de-Castro CGS. Assistência farmacêutica e acesso a medicamentos. Rio de Janeiro: Fiocruz; 2007.
11. Nascimento Junior JM, Alexandre RF, Costa LH, Santana RS, Lupatini EO, Domingues PHF et al. Avanços e perspectivas da RENAME após novos marcos legais: o desafio de contribuir para um SUS único e integral. Rev Eletrôn Gestão Saúde. 2015[acesso $10 \mathrm{dez}$ 2015];6(Supl 4):3354-71. Disponível em: http://rebrats.saude.gov.br/images/noticias/ArtigoRename.pdf

12. Cartaxo RJA, Antunes AMS, Magalhães JL. Produção de fármacos e medicamentos no Brasil: uma proposta de metodologia para priorização da Lista Estratégica no Âmbito do SUS. RECIIS. RECIIS Rev Eletrônica Comun Inf Inov Saúde. 2014[acesso 10 dez 2015];8(4):478-95. Disponível em: http://www.reciis.icict.fiocruz.br/index. $\mathrm{php} / \mathrm{reciis} /$ article/view/434

13. Ministério da Saúde (BR). Portaria $n^{\circ} 2.531$, de 12 de novembro de 2014. Redefine as diretrizes e os critérios para a definição da lista de produtos estratégicos para o Sistema Único de Saúde (SUS) e o estabelecimento das Parcerias para o Desenvolvimento Produtivo (PDP) e disciplina os respectivos processos de submissão, instrução, decisão, transferência e absorção de tecnologia, aquisição de produtos estratégicos para o SUS no âmbito das PDP e o respectivo monitoramento e avaliação. Diário Oficial União. 13 nov 2014.

14. Figueiredo T A. Assistência farmacêutica no Sistema Único de Saúde e a carga de doença no Brasil [tese]. Rio de Janeiro: Escola Nacional de Saúde Pública Sergio Arouca; 2015.

15. Ministério da Saúde (BR). Portaria $n^{\circ} 1$, de 2 de janeiro de 2015. Estabelece a Relação Nacional de Medicamentos Essenciais - RENAME 2014 no âmbito do Sistema Único de Saúde (SUS). Diário Oficial União. 3 jan 2015.

16. Ministério da Saúde (BR), Secretaria de Ciência, Tecnologia e Insumos Estratégicos, Departamento de Assistência Farmacêutica e Insumos Estratégicos. Relação Nacional de Medicamentos Essenciais: RENAME 2014. 9a ed. Brasília, DF: Ministério da Saúde; 2015.

17. Portal Fiocruz. HIV/Aids: Fiocruz dá novo passo para a produção do antirretroviral Atazanavir. Rio de Janeiro: Fundação oswaldo Cruz; 2014[acesso 9 jul 2014]. Disponível em: https://portal.fiocruz.br/pt-br/content/instituto-detencologia-em-farmacos-produzira-sulfato-de-atazanavir

18. Ministério da Saúde (BR). Portaria $n^{\circ} 3.089$, de 11 de dezembro de 2013. Redefine a lista de produtos estratégicos para o Sistema Único de Saúde (SUS) e as respectivas regras e critérios para sua definição. Diário Oficial União. 12 dez 2013. 
19. Grupo de Trabalho sobre Propriedade Intelectual - GTPI. Casos: Brasil. Rio de Janeiro: Grupo de Trabalho sobre Propriedade Intelectual; 2015[acesso 7 jul 2014]. Disponível em: http://www.deolhonaspatentes.org.br/media/ Contrato\%20-\%20Atazanavir.pdf

20. Brasil. Lei $n^{\circ} 6.360$, de 23 de setembro de 1976. Dispõe sobre a Vigilância Sanitária a que ficam sujeitos os medicamentos, as drogas, os insumos farmacêuticos e correlatos, cosméticos, saneantes e outros produtos, e dá outras providências. Diário Oficial União. 24 set 1976.

21. Agência Nacional de Vigilância Sanitária - Anvisa. Resolução - RDC n 136, de 29 de maio de 2003. Dispõe sobre o registro de medicamento novo. Diário Oficial União. 2 jun 2003.

22. U.S. Department of Health and Human Services, U.S. Food and Drug Administration. Development \& approval process (drugs). Silver Spring: U.S. Food and Drug Administration; 2014[acesso 7 jul 2014]. Disponível em: http://www.fda. gov/Drugs/DevelopmentApprovalProcess/default.htm

23. Gava CM. Registro sanitário de medicamentos novos: as normas legais e uma análise do mercado brasileiro [dissertação]. Rio de Janeiro: Escola Nacional de Saúde Pública Sergio Arouca; 2005.

24. European Medicines Agency - EMA. The European regulatory system for medicines and the European Medicines Agency: a consistent approach to medicines regulation across the Euopean Union. London; 2014[acesso 21 dez 2014]. Disponível em: http://www.ema.europa.eu/docs/en_GB/ document_library/Brochure/2014/08/WC500171674.pdf

25. Kashyap UN, Gupta V, Raghunandan HV. Comparison of drug approval process in United States \& Europe. J Pharm Sci Res. 2013;5(6):131-6.

26. Downing NS, Aminawung JA, Shah ND, Braunstein JB, Krumholz HM, Ross JS. Regulatory review of novel therapeutics: comparison of three regulatory agencies. $\mathrm{N}$ Engl J Med. 2012;366(24):2284-93. doi: 10.1056/NEJMsa1200223

27. Corporação Nacional de Incorporação de Tecnologia no SUS - Conitec. Relatório de Recomendação: protocolo clínico e diretrizes terapêuticas: prevenção da transmissão vertical de HIV, sífilis e hepatites virais: relatório de recomendação. [S.l.]: Corporação Nacional de Incorporação de Tecnologia no SUS; 2015[acesso 19 dez 2015]. Disponível em: http: // conitec.gov.br/images/Consultas/Relatorios/2015/ Relatorio_PCDT_TransmissaoVertical_CP.pdf

28. Ministério da Saúde (BR), Secretaria de Vigilância em Saúde, Programa Nacional de DST e Aids. Recomendações para terapia anti-retroviral em adultos e adolescentes infectados pelo HIV 2004. Brasília, DF: Ministério da Saúde; 2005[acesso 19 nov 2015]. Disponível em: http://bvsms. saude.gov.br/bvs/publicacoes/06consenso.pdf

29. Ministério da Saúde (BR), Secretaria de Vigilância em Saúde, Programa Nacional de DST e Aids. Recomendações para terapia anti-retroviral em adultos e adolescentes infectados pelo HIV 2005/2006. Brasília, DF: Ministério da Saúde; 2007[acesso 19 nov 2015]. Disponível em: http://bvsms.saude.gov.br/bvs/publicacoes/consenso_ adulto_2005_2006.pdf
30. Carter M, ed. Anti-Hiv drugs. 10a ed. [S.l.]: nam www. aidsmap.com; 2010[acesso 27 nov 2015]. Disponível em: http://www.aidsmap.com/files/file1000883.pdf

31. Australian Government, Department of Health. The Pharmaceutical Benefits Scheme - PBS. [S.I.]: Department of Health; 2015[acesso 27 nov 2015]. Disponível em: http://www.pbs.gov.au/info/about-the-pbs

32. Thomson Micromedex. Drugdex System 2015. [S.I.]: Truven Health Analytics; 2015[acesso 15 nov 2015]. Disponível em: http://www.micromedexsolutions. com/micromedex2/librarian/ND_T/evidencexpert/ ND_PR/evidencexpert/CS/CA0E5F/ND_AppProduct/ evidencexpert/DUPLICATIONSHIELDSYNC/74638D/ ND_PG/evidencexpert/ND_B/evidencexpert/ ND_P/evidencexpert/PFActionld/ pf. HomePage?navitem=topHome\&isToolPage=true

33. Chaves GC, Hasenclever L, Osorio-de-Castro CGS, Oliveira MA. Estratégias de redução de preços de medicamentos para aids em situação de monopólio no Brasil. Rev Saúde Pública. 2015;49:86. doi:10.1590/S0034-8910.2015049005459

34. World Health Organization - WHO. The selection and use of essential medicines: Report of the WHO Expert Committee, 2013 (including the 18th WHO Model List of Essential Medicines and the 4th WHO Model List of Essential Medicines for Children). Geneva: World Health Organization; 2013[acesso $18 \mathrm{dez}$ 2015]. (WHO technical report series, vol 985). Disponível em: http://apps.who. int/iris/bitstream/10665/112729/1/WHO_TRS_985_eng. pdf?ua $=1$

35. Médecins Sans Frontières - MSF. Generic competition pushing down HIV drug prices, but patents keep newer drugs unaffordable. Geneva: Médecins Sans Frontières; 2013[acesso 18 dez 2015]. (Press release Report, 2 july 2013). Disponível em: http://www.who.int/selection_ medicines/committees/expert/19/applications/MSF_ Comments.pdf?ua $=1$

36. Medicines Patent Pool - MPP. License and technology transfer agreement: Bristol-Myers Squibb Company. Geneva: Medicines Patent Pool; 2013[acesso 18 dez 2015]. Disponível em: http://www.medicinespatentpool.org/wp-content/ uploads/MPP-License-and-technology-transfer-agreementSigned22.pdf

37. Corrêa $M$, Cassier $M$, editores. Aids e saúde pública: contribuições à reflexão sobre uma nova economia política do medicamento no Brasil. Rio de Janeiro: EdUERJ; 2010. Saúde pública, ciência e indústria: os programas industriais de cópia de medicamentos contra Aids em laboratórios farmacêuticos brasileiros e a política de acesso universal ao tratamento; p. 141-76.

38. Chaves GC. Interfaces entre a produção local e o acesso a medicamentos no contexto do Acordo TRIPS da Organização Mundial do Comércio [tese]. Rio de Janeiro: Escola Nacional de Saúde Pública Sergio Arouca; 2016.

39. Brasil. Lei $n^{\circ} 9.279$, de 14 de maio de 1996. Regula direitos e obrigações relativos à propriedade industrial. Diário Oficial União. 15 maio 1996. 
40. Barroso WBG. Procedimento de oposição: o caso Tenofovir. In: Possas C, Larouzé B, editores. Propriedade intelectual e políticas públicas para o acesso aos antirretrovirais nos países do Sul. Rio de Janeiro: E-papers; 2013.
41. Nascimento Junior JM. Compulsory licensing of efavirenz in Brazil. In: Pharmaceuticals Rio Meeting; 23 fev 2010[acesso 4 dez 2014]. Disponível em: http://www.accesstopharmaceuticals.org/ case-studies-in-global-health/efavirenz-brazil/

Conflito de Interesse

Os autores informam não haver qualquer potencial conflito de interesse com pares e instituições, políticos ou financeiros deste estudo.

(ब) (1) (

Esta publicação está sob a licença Creative Commons Atribuição 3.0 não Adaptada.

Para ver uma cópia desta licença, visite http://creativecommons.org/licenses/by/3.0/deed.pt_BR. 